\title{
Diffusional Motion as a Gauge of Fluidity and Interfacial Adhesion.
}

\section{Supported Alkylphosphonate Monolayers}

\author{
S. M. Baumler, Thomas M. Reidy, and G. J. Blanchard* \\ Michigan State University, Department of Chemistry \\ 578 S. Shaw Lane \\ East Lansing, MI 48824 USA
}

\begin{abstract}
We report on the use of diffusion measurements to gauge the fluidity and surface binding properties of a molecular monolayer. The monolayer film consists of octadecyl1-phosphonic acid (ODPA) and controlled amounts of a lyso-phosphatidic acid tagged with the fluorescent probe BODIPY (BLPA). The monolayer films were formed using a Langmuir-Blodgett (LB) trough and deposited onto a glass slide. Monolayer morphology was characterized during film formation using Brewster angle microscopy (BAM). Fluorescence Recovery After Photobleaching (FRAP) microscopy was used to measure translational diffusion of BLPA and Fluorescence Anisotropy Decay Imaging (FADI) was used to measure rotational diffusion of the BLPA chromophore. These results provide information on the motional freedom of the probe and, importantly, on the strength of interaction between the probe and the support. Compositional variations in the monolayer give rise to changes in constituent dynamics that reflect intermolecular interactions.
\end{abstract}

Keywords: Layer adhesion, FRAP, Fluorescence Anisotropy Decay Imaging, LangmuirBlodgett Films

\footnotetext{
*Author to whom correspondence should be addressed. Email: blanchard@ chemistry.msu.edu. Email addresses: SMB: baumler@chemistry.msu.edu, TR: Thomas.reidy@kings.edu. Tel: +0115173559715 x224, fax +0115173531793.
}

\section{$-2-$}

(C) 2016. This manuscript version is made available under the Elsevier user license http://www.elsevier.com/open-access/userlicense/1.0/ 


\section{Introduction}

Monolayer films have been examined extensively over the past half-century because of their ability to alter the properties of an interface for a desired purpose. A single layer of molecules can be used to change the polarity of a surface, or to mediate electron transfer, for example. With technology requiring ever smaller dimensions, it is important to gain molecular-level control over not only the chemical identity of interfacial layers, but also to be able to control their physical properties.[1] We have undertaken the examination of a representative interfacial monolayer with the aim of quantitating the fluidity and adhesion of the adlayer to the interface. Understanding interfacial dynamics is a prerequisite to controlling these properties.

One family of monolayer films that has received extensive research attention is self-assembled monolayers (SAMs) of alkanethiols on gold.[2-13] That work has demonstrated the delicate balance of forces required to produce a monolayer and at the same time many practical applications have stemmed from this chemical system, including patterned interfaces for lithographic and other applications.[14-17] In addition to the alkanethiol SAMs, Langmuir-Blodgett (LB) films have enjoyed wide use.[18-21] The interactions between the amphiphilic film constituents and the supporting surface are fundamentally different for the two systems, with physical robustness favoring alkanethiol SAMs.[12, 13] LB films, however, allow for greater control over composition and ordering, and also produce films that exhibit controllable mobility.[22] This latter feature is important especially in cases where films benefit from defect mitigation or the ability to adapt to interfacial features. Both approaches to monolayer film formation allow complex molecules to form well-ordered two-dimensional 
assemblies. For both systems, an understanding of the interrelationship between the nanoscale heterogeneities at the support surface and defects within the film itself, and their effect(s) on the long-range order of the films, remains to be developed fully.

Previous work by the Talham group has shown that films of considerable ordering and structure can be prepared using LB methodology using organophosphonic acids with divalent metals at the appropriate subphase $\mathrm{pH} .[23,24]$ We are concerned with the introduction of structural heterogeneities into an amphiphilic monolayer structure, and how the film dynamics and organization change with the amount of "defect" species present. We use octadecylphosphonic acid (ODPA) as the amphiphile for the formation of the LB film and a fluorophore-tagged lysophospholipid (BLPA) as the dissimilar defect molecule. Because BLPA contains the BODIPY chromophore, it also serves as the optical probe in this work. We have chosen to use phosphonate LB films in this work because, ultimately, there is greater structural versatility and range of dynamics available with phosphonates than the corresponding carboxylates.[23-29]

We use the BLPA probe to measure the diffusional properties on both short (molecular) and long $(\mu \mathrm{m})$ length scales using rotational- and translational-diffusion measurements, respectively. Comparison of these two physical quantities affords quantitative information on the role of the defect molecule in mediating organization within the film and on the strength of interaction between the film and the support. While the study of dynamics in LB and related films is not new,[22, 30-34] our approach to comparing diffusional processes across a range of length scales is both novel and information-rich. We find that the forces binding the adlayer to the interface exceed those expected for simple physisorption, and more closely approximate the strength of 
hydrogen bond enthalpies in water. The ability to quantitate such interactions opens the door to better understanding how to control the properties of supported monolayer films and, simultaneously, provides direct information on the molecular motion that characterizes these interfaces.

\section{Materials and Methods}

Materials. Octadecylphosphonic acid (ODPA) $\left(\mathrm{C}_{18} \mathrm{H}_{39} \mathrm{O}_{3} \mathrm{P}, 97 \%\right.$ purity), barium chloride dihydrate $\left(\mathrm{BaCl}_{2} \cdot 2 \mathrm{H}_{2} \mathrm{O}\right.$, Reagent Grade), and tetrahydrofuran (THF, 99.9\%) were obtained from Sigma Aldrich and used as received. The chromophore used in these studies was 1-(12-[4-(dipyrrometheneboron difluoride) butanoyl] amino) dodecanoyl-2hydroxy-sn-glycero-3-phosphate (ammonium salt) (BLPA) (TopFluor Lyso PA, >99\%, Avanti Polar Lipids). Water (18 M $\Omega-\mathrm{cm})$ was obtained from a Milli-Q Plus water purification system and used for all experiments.

Langmuir Monolayer Preparation and LB Film Deposition. The barium ODPA (Ba-ODPA) LB films were prepared by the Langmuir-Blodgett technique.[18-20] We used a large Teflon ${ }^{\circledR}$ Langmuir trough (KSV Nima) equipped with a dipping mechanism and a platinum Wilhelmy plate attached to a balance with an automated feedback system to maintain a constant surface pressure during film transfer. Herein, we use the term 'Langmuir monolayer' to refer to a monolayer at the air-water interface and LB film to refer to the monolayer transferred to the solid support.

The glass substrates were standard microscope slides $(1 \mathrm{~mm} \times 25 \mathrm{~mm} \times 75 \mathrm{~mm}$, Globe Scientific) and were placed in a piranha solution $\left(1 \mathrm{H}_{2} \mathrm{O}_{2}: 3 \mathrm{H}_{2} \mathrm{SO}_{4}\right.$, Caution! Strong oxidizer!) for $30 \mathrm{~min}$ prior to transfer to the LB-trough for dipping. After cleaning, the substrates were rinsed with water and placed in a covered beaker containing water until 
they were used for deposition. To produce stable monolayers the subphase was a $5 \mathrm{mM}$ $\mathrm{BaCl}_{2}$ aqueous solution. The subphase $\mathrm{pH}$ was measured to be 5.4. The temperature of the subphase was maintained at $23^{\circ} \pm 0.1^{\circ} \mathrm{C}$ using a circulating water bath (Fisher) attached to the trough. For each dipping experiment $150 \mu \mathrm{L}$ of a $1 \mathrm{mg} / \mathrm{mL}$ ODPA in THF solution was used for monolayer spreading. Solutions containing 5 mol\% BLPA (BLPA5) and 10 mol\% BLPA (BLPA-10) were prepared by mixing appropriate amounts of the ODPA solution and $1 \mathrm{mg} / \mathrm{mL}$ BLPA in THF solution and mixing for $30 \mathrm{sec}$ by hand. The amount of solution spread on the surface was calculated to give an initial surface pressure $\pi$ below $1 \mathrm{mN} / \mathrm{m}$. During spreading, care was taken to prevent the spreading needle from touching the surface as well as preventing the surface pressure $\pi$ from rising above 0.5 $\mathrm{mN} / \mathrm{m}$ in order to ensure complete spreading. After spreading, the monolayer was allowed to equilibrate for $30 \mathrm{~min}$ to ensure solvent evaporation and monolayer relaxation, before the barriers were moved inward at $5 \mathrm{~mm} / \mathrm{min}$ (mean molecular area compression of $<1 \AA^{2} / \mathrm{min}$ ) to compress the surface monolayer to the pressure used for dipping (30 $\mathrm{mN} / \mathrm{m}$ ). The compressed monolayer was allowed to equilibrate for 15 minutes before it was transferred onto the glass slide. The monolayer was transferred (upstroke) at a dipping speed of $5 \mathrm{~mm} / \mathrm{min}$ while keeping the pressure constant, covering a total area of $35 \mathrm{~mm} \times 25 \mathrm{~mm}$ on each side of the glass slide. The coated slides were dried in air for at least $1 \mathrm{hr}$. before FRAP and FADI measurements were made.

Brewster angle microscopy. We characterize the morphology of the monolayers formed on the LB-trough using a Brewster angle microscope (BAM, Accurion UltraBAM). This instrument is characterized by $2 \mu \mathrm{m}$ spatial resolution and an adjustable angle of incidence. The laser wavelength used for BAM measurements is 653 
$\mathrm{nm}$ (diode laser) and the instrument is operated using software supplied by the manufacturer.

Steady State Fluorescence Measurements. Excitation and emission spectra of the BLPA chromophore in THF was collected using a Jobin Yvon Spex Fluorolog-3 spectrometer. Emission spectra were collected for BLPA in THF as well as the $5 \mathrm{~mol} \%$ and $10 \mathrm{~mol} \%$ samples to verify the presence and band positions of the incorporated BLPA chromophore. Spectral resolution was set to $1 \mathrm{~nm}$ for both the excitation and emission monochromators for all measurements.

Optical ellipsometry. Optical ellipsometry measurements were performed to determine LB film thickness using a J. A. Woollam Co., Inc. model EC270 spectroscopic ellipsometer with a fixed angle $\left(75^{\circ}\right)$ and wavelength range of 400-1100 $\mathrm{nm}$. The EC270 is equipped with $75 \mathrm{~W}$ Xenon lamp source and diode array detector to simultaneously detect 44 wavelengths. The LB films were prepared on single crystal (100) silicon wafers (University Wafer, Boston, MA). The fitting for the ODPA layer thickness was accomplished by modeling the silicon substrate as adlayers of silicon dioxide with fixed thickness and refractive index of 1.46 , and a generic organic film layer with a refractive index of 1.5. The silicon dioxide adlayer was determined on a freshly cleaned substrate and measured to be $17 \pm 1 \AA$.

Fluorescence Recovery After Photobleaching (FRAP) Measurements. FRAP measurements were conducted on a Nikon $\mathrm{C}^{+}$confocal laser scanning microscopy system consisting of a Nikon Eclipse Ti-E inverted microscope with a confocal scanning system (Nikon Ti-S-CON). The BLPA was excited with the $488 \mathrm{~nm}$ line of an $\mathrm{Ar}^{+}$laser (Nikon Lu-N4), and emission was detected at $525 \mathrm{~nm}$ using a standard PMT detector 
(Nikon C2-DU3). A 10x objective lens was used for the LB films. Initial intensities $\left(I_{0}\right)$ were measured for a minimum of $10 \mathrm{~s}$. The bleaching time was set to $5 \mathrm{~s}$, and the recovery signal after photobleaching was collected over a minimum of 6 min. The recovery signals, $I_{\text {frap }}(t)$, were normalized using Eq. 1, where $I_{\text {bleach }}$ is the intensity immediately following photobleaching, $I_{0}$ is the maximum intensity during the $15 \mathrm{~s}$ prior to photobleaching, and $I_{\text {norm }}(t)$ is the calculated normalized intensity.

$$
I_{\text {norm }}(t)=\frac{I_{\text {frap }}(t)-I_{\text {bleach }}}{I_{0}-I_{\text {bleach }}}
$$

A freshly prepared LB film was placed above the objective and approximately $100 \mu \mathrm{L}$ of milliQ water was added to a random location of the film. Five measurements were made within the water overlayer on each side of a slide, representing a total of 10 measurements per slide, over 6 slides. The translational diffusion constant $\left(D_{T}\right)$ and percent mobility were obtained by fitting the normalized data to the Ellenberg equation (Eq. 2),[35]

$I(\mathrm{t})=\mathrm{I}_{\text {final }}\left[1-\left(\frac{w^{2}}{w^{2}+4 \pi D_{T} t}\right)^{1 / 2}\right]$

$I_{\text {final }}$ represents the fluorescence intensity in the photobleached region at times greater than $5 \mathrm{~min}, w$ is the diameter of the photobleached spot $(10 \mu \mathrm{m})$, and $D_{T}$ is the translational diffusion constant $\left(\mu \mathrm{m}^{2} / \mathrm{s}\right)$. Due to normalization method (Eq. 1), $I_{\text {final }}$ is the fractional mobility of BLPA in the LB film. The recovery time for the photobleach $\left(\tau_{1 / 2}\right)$ is defined as the time it takes for the depletion recovery to reach $50 \%$ of the final intensity (Eq. 3), 


$$
\tau_{1 / 2}=\frac{0.75 w^{2}}{\pi D_{T}}
$$

Fluorescence Anisotropy Decay Imaging (FADI) Measurements. The instrument used to acquire fluorescence anisotropy decay images has been described previously.[36] Briefly, the instrument is based on an inverted microscope optical configuration (Nikon Eclipse Ti-U). All images were collected using a 10x microscope objective. Timeresolved data collection is achieved using the confocal scanning unit (Becker \& Hickl DCS-120) attached to an output port of the microscope and is controlled by a galvoscanning drive unit (Becker \& Hickl GDA- 120). The confocal scanning unit (DSC-120) is equipped with a polarizing beam splitter and two avalanche photodiode detectors (IDQuantique ID100). Polarized fluorescence transients are acquired using time-correlated single photon counting (TCSPC) detection electronics (Becker \& Hickl SPC-152, PHD$400 \mathrm{~N}$ reference diode) and were used to determine the induced orientational anisotropy decay function (Eq. 4).

The light source for these experiments was a synchronously pumped cavity dumped dye laser (Coherent 702) excited by the output of a passively mode locked Nd: $\mathrm{YVO}_{4}$ laser (Spectra Physics Vanguard). The dye laser is cavity dumped (Gooch and Housego 64389.5-SYN-9.5-1 cavity dumper driver) to control the repetition rate. The dye laser output is characterized by ca. 5 ps pulses at a repetition rate of $4 \mathrm{MHz}(250 \mathrm{~ns}$ interpulse spacing). For this work the dye laser output was set to $490 \mathrm{~nm}$ using Stilbene 420 dye (Exciton) and the emission collection window was $535 \pm 30 \mathrm{~nm}$, determined by a band pass filter in the confocal scanning unit. These wavelengths were selected based on the excitation and emission spectra of the BLPA chromophore (Fig. 4). 
Supported LB films were placed above the microscope objective and approximately $100 \mu \mathrm{L}$ of Milli-Q water was added to a random location of the film. Two measurements were made within the hydrated region on each side of the slide, representing a total of 4 measurements per slide over 4 slides. Each measurement produced a 256x256 pixel $^{2}$ image with 256 time points from both the parallel and perpendicular channels. The images were binned to average the data over a 4 pixel $\times 4$ pixel region. Anisotropy decay time constants were obtained by fitting the induced anisotropy decay equation, which is calculated from the averaged polarized transients (Eq. 4):

$R(t)=\frac{I_{\|}(t)-I_{\perp}(t)}{I_{\|}(t)+2 I_{\perp}(t)}$

The anisotropy decay time constant $\left(\tau_{\mathrm{HR}}\right)$ or can be determined by fitting $R(t)$ to Eq. 5 , which is derived from the hindered rotor model.[37, 38]

$$
R(t)=R(\infty)+(R(0)-R(\infty)) \exp \left(-t / \tau_{H R}\right)
$$

The terms $R(0)$ and $R(\infty)$ are the zero- and infinite-time anisotropies, respectively, and the term $\tau_{\mathrm{HR}}$ is the anisotropy decay time constant. We consider the physical significance of these data below.

\section{Results and Discussion}

We are concerned with the fundamental properties of LB films. These include the stability and fluidity of the film as a function of how it was formed, and the role that probe molecules may play in mediating film organization and dynamics. We use Langmuir isotherms $(\pi / \mathrm{A})$ to examine changes in the monolayer density as a function of applied pressure, ionic strength of the subphase, and the addition of selected amounts of 
BLPA. We use a suite of optical techniques to evaluate the morphology (BAM) and dynamics (FADI, FRAP) of the LB films as a function of system conditions. We consider the information content of these data below, focusing first on the role of subphase composition on Langmuir monolayer properties.

Effect of subphase ionic strength on ODPA Langmuir monolayer morphology.

Surface-pressure vs. area isotherms ( $\pi / \mathrm{A}$-isotherms) have been acquired for monolayers prepared over an aqueous subphase containing no ionic constituents and a subphase containing $5 \mathrm{mM} \mathrm{BaCl}_{2}$ (Fig. 2). BAM images were collected at $5 \mathrm{mN} / \mathrm{m}$ surface pressure increments for each sample. The isotherm for the monolayer formed on the water subphase consists of a sharp increase in pressure at a mean molecular area of $21 \AA^{2}$ and a characteristic collapse of the monolayer at ca. $62 \mathrm{mN} / \mathrm{m}$. For the subphase containing 5 $\mathrm{mM} \mathrm{BaCl}$, the $\pi / \mathrm{A}$-isotherm takes a different form. The isotherm does not begin to exhibit a significant increase in pressure until ca. $18 \AA^{2} /$ molecule, has a mean molecular area of $13 \AA^{2}$, and the slope is more shallow $\left(11 \mathrm{mN} / \mathrm{m} / \AA^{2}\right)$ compared to the isotherm of the monolayer formed on the water subphase $\left(16 \mathrm{mN} / \mathrm{m} / \AA^{2}\right)$. Additionally, the $\mathrm{Ba}^{2+}$ containing monolayer does not exhibit a collapse at high pressure similar to the monolayer formed on the water subphase. Rather, the monolayer formed on the $\mathrm{BaCl}_{2}$ containing subphase maintains a constant pressure at ca. $60 \mathrm{mN} / \mathrm{m}$ with decreasing surface area. BAM images of the two monolayers reveal differences beginning at formation, before any compression occurs (Figs. 2B, C). The monolayer formed on the water surface exhibits fluid regions with small domain ordering present as the white elliptical/circular regions with smaller holes throughout. The monolayer formed with $\mathrm{BaCl}_{2}$ in the subphase exhibits larger island-like regions with dark domain boundaries. 
Upon compression of the monolayer on the water subphase, the images appear uniform with small darker lines throughout, up to the point of collapse, when bright lines and cracks begin to appear (Figs. 2D-G). The monolayer formed on the subphase containing $\mathrm{BaCl}_{2}$ exhibits scattered bright islands, which do not appear to change in size and/or shape upon compression; these island regions appear to reorganize to fill in the liquid regions with increasing pressure. The Talham group has shown previously that the formation of crystalline $\mathrm{Ba} / \mathrm{ODPA}$ monolayers is highly $\mathrm{pH}$ dependent, and can be formed around $\mathrm{pH}$ 8-9.[23] We attribute much of the heterogeneity within these BAM images to be due the subphase $\mathrm{pH}$ being lower than the optimal $\mathrm{pH}$ for deposition. Ellipsometric thickness measurements after deposition onto the glass slides confirm thicknesses consistent with a monolayer $(27 \pm 1 \AA$ )..[29]

The introduction of ionic character into the subphase allows for two phenomena to occur. Increasing ionic strength is known to stabilize the condensed phase allowing for the crystal structure of the long-chain aliphatic acids to be maintained during transfer to the glass support.[39] Through charge compensation, the $\mathrm{Ba}^{2+}$ cation would allow the negatively charged polar phosphonic acid headgroups to pack more closely than they would in water at a $\mathrm{pH}$ above the $\mathrm{pK}_{\mathrm{a}}(\mathrm{s})$ of the headgroup. Due to the change in the headgroup spacing, it is expected that the tilt angle of the aliphatic chains will be different for deposition on the two subphases,[40] and our experimental findings are consistent with this expectation.

Effect of Increasing BLPA Concentration on ODPA Langmuir monolayer Morphology. $\pi / \mathrm{A}$-isotherms have also been acquired for monolayers prepared over an aqueous subphase containing $5 \mathrm{mM} \mathrm{BaCl}_{2}$ at two different BLPA concentrations (Fig. 3). 
BLPA-5 increases the mean molecular area of pure ODPA from $13 \AA^{2}$ to $25 \AA^{2}$. For BLPA-10 the mean molecular area decreases from $25 \AA^{2}$ for BLPA-5 to $17 \AA^{2}$. The slopes of the isotherm curves increase with increasing BLPA concentration, from 11 $\mathrm{mN} / \mathrm{m} / \AA^{2}$ for pure ODPA, to $13 \mathrm{mN} / \mathrm{m} / \AA^{2}$ for BLPA-5, and $20 \mathrm{mN} / \mathrm{m} / \AA^{2}$ for BLPA-10. The decrease in mean molecular area and increase in slope correlated with the increase in BLPA concentration can be explained in the context of an increased packing density of the BLPA through phase segregation.

The mean molecular areas derived from the data shown in Fig. 3 were found to be lower than those reported previously.[23] We attribute the rise of the isotherms at values lower than expected to several possible causes. The first is due to the solvent system used during application of the monolayer. ODPA was found to dissolve in THF at concentrations as high as $1 \mathrm{mg} / \mathrm{mL}$ and BLPA also exhibited the requisite solubility in this solvent. As a consequence of this solvent choice, the miscibility of the subphase with THF gave rise to the loss of a finite amount of material at the subphase surface during deposition, leading to lower than expected mean molecular areas (Fig. S1). The second possible reason is due to the solubility of the BLPA chromophore in the subphase at increasing pressures (Fig. 2A). When comparing the pure BLPA on water (Fig. 2A, iii.) to pure BLPA on the barium chloride sub-phase (Fig. 2A, iv.), the addition of ionic character improves the formation of a stable monolayer slightly, likely by decreasing the solubility of the BLPA.

BAM images of the monolayer without BLPA formed with $\mathrm{BaCl}_{2}$ in the subphase are shown in Fig. 2. The addition of 5\% BLPA creates two distinct regions (Figs. 3B, C). Upon compression, both BLPA-5 and BLPA-10 exhibit bright cracks at the collapse 
pressure (Figs. 3F, G). BLPA-5 contains $c a .50 \%$ more bright regions than BLPA-10, and the bright regions of the BLPA-5 are, on average, $c a$. $20 \%$ smaller than those in BLPA-10. We believe these bright spots are aggregates of BLPA dispersed throughout the monolayer and the differences in quantity of bright regions correlates well with the two-fold increase in amount of BLPA present.

Formation of Aggregates at Higher BLPA Concentrations. The steady state absorbance and emission spectra of BLPA in THF, as well as the emission for BLPA-5 and BLPA-10 LB films are shown in Fig. 4A. Increasing the concentration of BLPA in the LB films causes a bathochromic shift of the emission spectrum of $\sim 6 \mathrm{~nm}$. The difference in band shift between the BLPA-5 and BLPA-10 films is $\sim 4 \mathrm{~nm}$. The BODIPY chromophore is known to form either $\mathrm{H}$ - or J- aggregates,[41-44] depending on the environment and functionalities attached to the BODIPY chromophore.[45-48] We attribute the slight bathochromic shift to the formation of J-aggregates in these films. The peak shift, whether bathochromic or hypsochromic, depends on the angle between the constituent chromophore transition dipole moments (Fig. 4B). J-aggregation is characterized by the angle between the constituent transition moments, $\gamma \leq 54.7^{\circ}$. As the angle between the BODIPY transition moments approaches zero, the J-aggregates are characterized by a head-to-tail orientation, while $\mathrm{H}$-aggregates are characterized by a transition moment angle $\gamma>54.7^{\circ}$, where the transition dipole moments are stacked faceto-face or there is considerable $\pi-\pi$ stacking in the aggregate. We attribute the observed spectral shift to aggregation, with the increased disorder induced by the BLPA-10 films decreasing the angle between the two chromophores and allowing for a more head-to-tail configuration. 
Steady state fluorescence images of BLPA-5 and BLPA-10 are shown in figure 4C and 4D, respectively. Increasing the concentration of BLPA in the LB films reveals an increase in pinhole defects and increased heterogeneity, evidenced by the bright striped regions the BLPA-10 image (Fig. 4D). The BLPA-5 image shows a considerable number of bright regions throughout the image. We attribute these bright spots to regions of BLPA aggregation. There is considerably less intense spotting in the BLPA-10 image. We attribute the decrease in spotting intensity to be due to two main factors. First, at higher concentrations the contrast between the BLPA aggregates and the larger film area decreases due to the overall increased presence of BLPA chromophore. Second, BLPA-5 shows larger regions of uniform intensity compared to BLPA-10 with alternating regions of high and low intensity (striping). This observation suggests not only that more BLPA is present, but BLPA may phase segregate into regions where the film exhibits different domain organization.

\section{Translational Diffusion Slowed by Aggregation at Increased BLPA}

Concentrations. Macroscopic translational diffusion of the ODPA/BLPA LB films was measured using FRAP. FRAP is sensitive to the organization of the LB film. FRAP data for both BLPA-5 and BLPA-10 films are shown in Fig. 5 and Table 1. The average translational diffusion constant of the BLPA-5 film is $0.26 \pm 0.09 \mu \mathrm{m}^{2} / \mathrm{s}$ with a mobile fraction of $16 \pm 4 \%$. The BLPA-10 film gives $\mathrm{D}_{\mathrm{T}}=0.09 \pm 0.02 \mu \mathrm{m}^{2} / \mathrm{s}$ with a mobile fraction of $23 \pm 4 \%$. The time constant for recovery of the BLPA-5 film is $90 \pm 30 \mathrm{~s}$ and for the BLPA-10 film $\tau=250 \pm 50 \mathrm{~s}$. These data were determined from the raw timescans by fitting to Eqs. 2 and 3. The three-fold decrease in both $\mathrm{D}_{\mathrm{T}}$ and $\tau$ indicate that translational motion of BLPA-10 is more hindered, and we assert that the reason for this 
is the aggregation of chromophores (vide infra). We consider the energetic implications of these findings below.

Rotational Diffusion and Information Content. FADI measurements have been performed for both BLPA-5 and BLPA-10 LB films (Table 2). Typical fluorescence anisotropy decays for the films are shown in Fig. 6. To gain greater insight into the effect of increased BLPA concentration on the surface structure and dynamics, we have acquired 256x256 pixel images of the fluorescence lifetime and anisotropy decay of the films (Figs. 6C-F). These data provide qualitative insight into how structural heterogeneity is induced at higher BLPA concentrations. BLPA-10 films have characteristically shorter average fluorescence lifetimes and longer anisotropy decay time constants. The shorter chromophore lifetime for the BLPA-10 films suggests quenching. We assert that the fluorescence quenching is due to the formation of BODIPY aggregates, consistent with the steady-state emission shift reported for these films (vide supra). The anisotropy decay images for the BLPA-10 films show both a broader distribution and longer anisotropy decay time constants than are seen for the BLPA-5 films. These data are consistent with chromophore aggregation at higher concentration.

To obtain quantitative information on the chromophore local environment, the images were analyzed as described above to obtain the average anisotropy decay time constant by fitting the collected fluorescence anisotropy decay transients to Eq. 5. The reorientation times for the films were $\tau_{\mathrm{HR}}=3,400 \pm 220 \mathrm{ps}$ and $4,000 \pm 200 \mathrm{ps}$ for the BLPA-5 and BLPA-10 films, respectively. The LB film under investigation here contains a tethered chromophore, so the hindered rotor model is appropriate (Eqs. 5 and 6 ). $[37,38]$ 


$$
\tau_{H R}=\frac{7 \theta_{0}^{2}}{24 D_{w}}
$$

$\theta_{0}=\cos ^{-1}\left(0.5\left[8\left(\frac{R(\infty)}{R(0)}\right)^{1 / 2}+1\right]^{1 / 2}-0.5\right)$

In this model, the (fast) chromophore motion is constrained by the medium surrounding it and its tether to the support surface. Rotational motion can be described as occurring in a cone of semi-angle $\theta_{0}$, and there will be a diffusive "wobbling" of the chromophore about its tethering bond. This motion is characterized by the quantity $\mathrm{D}_{\mathrm{w}}$. The relationship between $D_{w}$ and $D_{R O T}$ can be estimated by the approximation that the experimental $\tau \sim$ $\tau_{\mathrm{OR}} \sim \tau_{\mathrm{HR}}$, $\tau \approx \tau_{O R} \approx \tau_{H R}=\frac{1}{6 D_{R O T}}=\frac{7 \theta_{0}^{2}}{24 D_{w}}$ $D_{R O T}=\left(\frac{4}{7 \theta_{0}^{2}}\right) D_{w}$

The rotational diffusion constants were determined to be $\mathrm{D}_{\mathrm{ROT}}=49.0 \pm 3.1 \mathrm{MHz}$ and $\mathrm{D}_{\mathrm{ROT}}=41.8 \pm 2.1 \mathrm{MHz}$ for the BLPA-5 and BLPA-10 films, respectively (Eq. 7). The slower time constant in BLPA-10 is consistent with aggregation of the chromophores.

As can be seen in Eq. 7, the relationship between $D_{\text {ROT }}$ and $D_{w}$ depends on the angle of the confining cone (Fig. 7A). For $\theta_{0}=0$, the relationship is undefined, and this situation corresponds physically to the probe being immobile and unable to exhibit diffusional motion. For physically realistic angles, between $\mathrm{ca} .30^{\circ}$ and $90^{\circ}$, the coefficient $\left(4 / 7 \theta_{0}{ }^{2}\right)$ varies between 2.08 and 0.23 , for $\theta_{0}=54.7^{\circ}$, the magic angle, 0.63 , and for $\theta_{0}=43.4^{\circ}, D_{\text {ROT }}=D_{w}$. Owing to the heterogeneity in both the BLPA-5 and 
BLPA-10 films, the BLPA probe population is likely characterized by a random orientational distribution. For such a distribution the confining cone semi-angle distribution averages to $54.7^{\circ},[37,38]$ yielding a value of $\mathrm{D}_{\mathrm{ROT}}=0.63 \mathrm{D}_{\mathrm{w}}=30.9 \pm 2.0$ $\mathrm{MHz}$ for BLPA-5, and $26.5 \pm 1.3 \mathrm{MHz}$ for BLPA-10.

Implications of the Ba-ODPA/BLPA system thermodynamics on the diffusional dynamics. Comparing rotational diffusion and translational diffusion data on the same system can provide substantial insight into the properties of the system, with the detailed information content depending on the system. As noted above, chromophore motion is described in the context of the hindered rotor model, $[37,38]$ and it is the relationship of the wobbling diffusion constant, $\mathrm{D}_{\mathrm{w}}$, for chromophore motion about its tethering bond that we must relate to the rotational diffusion constant, $\mathrm{D}_{\mathrm{ROT}}$. Longer range, translational diffusion of the same chromophore within the LB film will be mediated by interactions between the amphiphile phosphonate headgroups and the support surface, and both of these issues have to be taken into account in extracting information from these data.

The relationship between rotational diffusion and translational diffusion is understood for a spherical particle is a uniform medium, where the rotational diffusion constant is given by the Debye-Stokes-Einstein equation (Eq. 8) and the translational diffusion constant is given by the Stokes-Einstein-Sutherland equation (Eq. 9).

$$
\begin{aligned}
& D_{R O T}=\frac{k_{B} T}{8 \pi \eta r^{3}} \\
& D_{T}=\frac{k_{B} T}{6 \pi \eta r}
\end{aligned}
$$

Where $\eta$ is the viscosity of the medium, $r$ is the radius of the diffusing particle and $\mathrm{k}_{\mathrm{B}} \mathrm{T}$ is the thermal energy term. There are well-established correction factors for Eq. 8 to 
account for the boundary condition between the particle and the medium in which it is immersed, as well as for the non-spherical shape of the particle, but those correction factors yield only small changes from Eq. 8 for the systems of interest, and their inclusion does not bear on the fundamental relationship between $\mathrm{D}_{\mathrm{ROT}}$ and $\mathrm{D}_{\mathrm{T}}$ (Eq. 10).

$$
D_{T}=\left(\frac{4 r^{2}}{3}\right) D_{R O T}
$$

Eq. 10 is frequently does not obtain experimentally because of the specific physical and chemical conditions of the system under investigation.

For the LB films reported here the amphiphile constituents interact with the solid support and it is possible to estimate the energy of that interaction. Experimentally, the value of $\mathrm{D}_{\mathrm{T}}$ recovered, $\mathrm{D}_{\mathrm{T}}{ }^{\text {eff }}$, is less than that expected if the adlayer were not interacting with the support (Eq. 9). There are a variety of ways to interpret a value of $\mathrm{D}_{\mathrm{T}}^{\text {eff }}$ less than that predicted by Eq. 9. While heterogeneities in the films certainly exist, including pinholes, regions of disproportionate ODPA:BLPA concentrations due to aggregation, or the potential formation of multilayer islands, the $\mathrm{D}_{\mathrm{T}}$ and $\mathrm{D}_{\mathrm{ROT}}$ values are acquired as average values over tens of microns of film surface. For a heterogeneous system such anomalous diffusion is considered to result from frustrated chromophore motion.[49-51] Since the film under consideration here contains only one amphiphile in addition to the chromophore, the notion of anomalous diffusion does not apply. For a system where there is an equilibrium between the free and surface-bound amphiphile, the value of $\mathrm{D}_{\mathrm{T}}{ }^{\text {eff }}$ reflects the fraction of time the chromophores are free to diffuse, i.e. not bound to the support. In this treatment we focus on the dynamic rather than the steady state (mobile fraction) contribution to the FRAP data. The steady state contributions to these data appear to be the same for the two chromophore concentrations to within the experimental 
uncertainty (Table 1), and the normalization of the experimental FRAP data can, in principle, influence the recovered value for the mobile fraction, where time-domain data are not affected by normalization.

We model this equilibrium according to Eq.11, with the diffusion constant of the bound species being zero.

$$
\begin{aligned}
& D_{T}^{\text {eff }}=A_{\text {free }} D_{T}+A_{\text {bound }} D_{T}^{\text {bound }}=A_{\text {free }} D_{T} \\
& A_{\text {free }}+A_{\text {bound }}=1 \\
& D_{T}^{\text {bound }}=0
\end{aligned}
$$

The free fraction, $A_{\text {free }}$, is related to the equilibrium constant for this system (Fig. 7B). If the bound amphiphiles have a surface coverage $\phi$, then the free sites are given by 1- $\phi$, which is equal to $A_{\text {free }}$, and the equilibrium constant for this system is

$$
K_{\text {eq }}=\frac{k_{\text {free }}}{k_{\text {bound }}}=\frac{(1-\phi)^{2}}{\phi}
$$

The fraction of free sites, $A_{\text {free }}$, is related to $\mathrm{K}_{\mathrm{eq}}$ by

$$
A_{\text {free }}=1-\left(\frac{\left(2+K_{e q}\right)-\sqrt{K_{e q}^{2}+4 K_{e q}}}{2}\right)
$$

Thus determination of $A_{\text {free }}$ from the experimentally-derived quantities $\mathrm{D}_{\mathrm{w}}, \theta_{0}$ and $\mathrm{D}_{\mathrm{T}}^{\text {eff }}$ provides $\mathrm{K}_{\mathrm{eq}}$.

We calculate the hydrodynamic volume of BLPA to be $602 \AA^{3}$, corresponding to $r$ $=5.2 \AA$ for a spherical diffusing species. Using Eq. 10 we calculate $\mathrm{D}_{\mathrm{T}}=11.1 \pm 0.7$ $\mu \mathrm{m}^{2} / \mathrm{s}$ for BLPA-5 and $\mathrm{D}_{\mathrm{T}}=9.6 \pm 0.5 \mu \mathrm{m}^{2} / \mathrm{s}$ for BLPA-10 using $\mathrm{D}_{\mathrm{ROT}}$ values derived from the experimental FADI data. 
The experimental values of $\mathrm{D}_{\mathrm{T}}^{\text {eff }}$ for BLPA, determined by FRAP, are $0.26 \pm 0.09$ $\mu^{2} / \mathrm{s}$ for BLPA-5 and $0.09 \pm 0.02 \mu \mathrm{m}^{2} / \mathrm{s}$ for BLPA-10. Using Eq. 11 we determine $A_{\text {free }}$ $=0.022 \pm 0.009$ for BLPA-5 and $0.009 \pm 0.002$ for BLPA-10. From $A_{\text {free }}=1-\phi$, we determine $\mathrm{K}_{\mathrm{eq}}=5.0(+4.9,-3.2) \times 10^{-4}$ for BLPA-5 and $8.2(+4,-3.3) \times 10^{-5}$ for BLPA-10. As the equilibrium constant for this system is cast as a dissociation constant, we can calculate the free energy of dissociation for the film constituents.

$$
\Delta G_{d i s}=-R T \ln K_{e q}
$$

For BLPA-5 we find $\Delta \mathrm{G}_{\mathrm{dis}}=18.5(+2.6,-1.7) \mathrm{kJ} / \mathrm{mol}$ and for BLPA-10, $\Delta \mathrm{G}_{\mathrm{dis}}=$ $22.9(+1.3,-0.9) \mathrm{kJ} / \mathrm{mol}$. There are several important pieces of information contained in these data. The first important point to note is that (dipolar) aggregation is more prominent for BLPA-10 than for BLPA-5, and the effective change in the net driving force for adlayer formation due to an unknown amount of aggregation is energetically favorable by $c a .4 \mathrm{~kJ} / \mathrm{mol}$. The second is that the strength of interaction between the phosphate amphiphile and the $\mathrm{Ba} / \mathrm{SiO}_{\mathrm{x}}$ surface is very similar to that of the hydrogen bond enthalpies of water (ca. $23 \mathrm{~kJ} / \mathrm{mol}$ ),[52] and consistent with previous results,[53] but somewhat larger than that reported for water in proximity of nonpolar species.[54] We attribute much of the observed interactions to be due to intermolecular hydrogen bonding between ODPA molecules or ODPA and the hydroxylated silica surface. The metal ions that were carried from the LB trough subphase may be facilitating interactions between the amphiphile and the support but these interactions cannot be considered complexation in any sense.[55-57] In fact, it is not likely that much interaction occurs between the $\mathrm{Ba}^{2+}$ and ODPA due to the sub-optimal $\mathrm{pH}$ during transfer to the support, $\mathrm{a}$ condition that reduces transfer of the metal ion to the support.[23] 

of the adlayer to the interface. By examining the diffusional behavior of this tethered chromophore over length scales ranging from molecular through macroscopic, we have demonstrated a direct and novel means for the evaluation of adlayer binding strength on a support surface. This is the first report that we are aware of that utilizes complementary molecular diffusion measurements for this purpose. Simultaneously, we are able to ascertain the motional freedom that is characteristic of this chromophore and the effect of chromophore aggregation on molecular motion. These data provide insight into the binding strength of a barium phosphonate species to a silica surface and, by extension, provide an estimate of the binding strength for related interfaces. The value obtained for $\Delta \mathrm{G}_{\mathrm{dis}}$, ca. $20 \mathrm{~kJ} / \mathrm{mol}$, is entirely consistent with interactions dominated by H-bonding[58] and there is also a chromophore concentration dependence that suggests some information on the interaction energy of aggregated chromophores.

We note that the strength of interaction for our monolayer with a silica support is very similar to the binding interactions responsible for the formation of alkanethiol monolayers on gold and mercury.[12, 13, 59] This is an important comparison to make because the spectroscopic evaluation of chromophore dynamics in an alkanethiol/gold SAM is limited by chromophore quenching,[60] and these data offer a way to at least estimate the expected dynamics for this family of systems. The next step in this work is to evaluate the role of chemical modification of the support as a means of controlling binding strength. 
Table 1: Translational diffusion constants $\left(\mathrm{D}_{\mathrm{T}}\right)$, Mobile fraction $\%$, and Recovery speed $\left(\tau_{1 / 2}\right)$ determined for the $5 \mathrm{~mol} \%$ and 10 mol\% substrates from FRAP measurements.

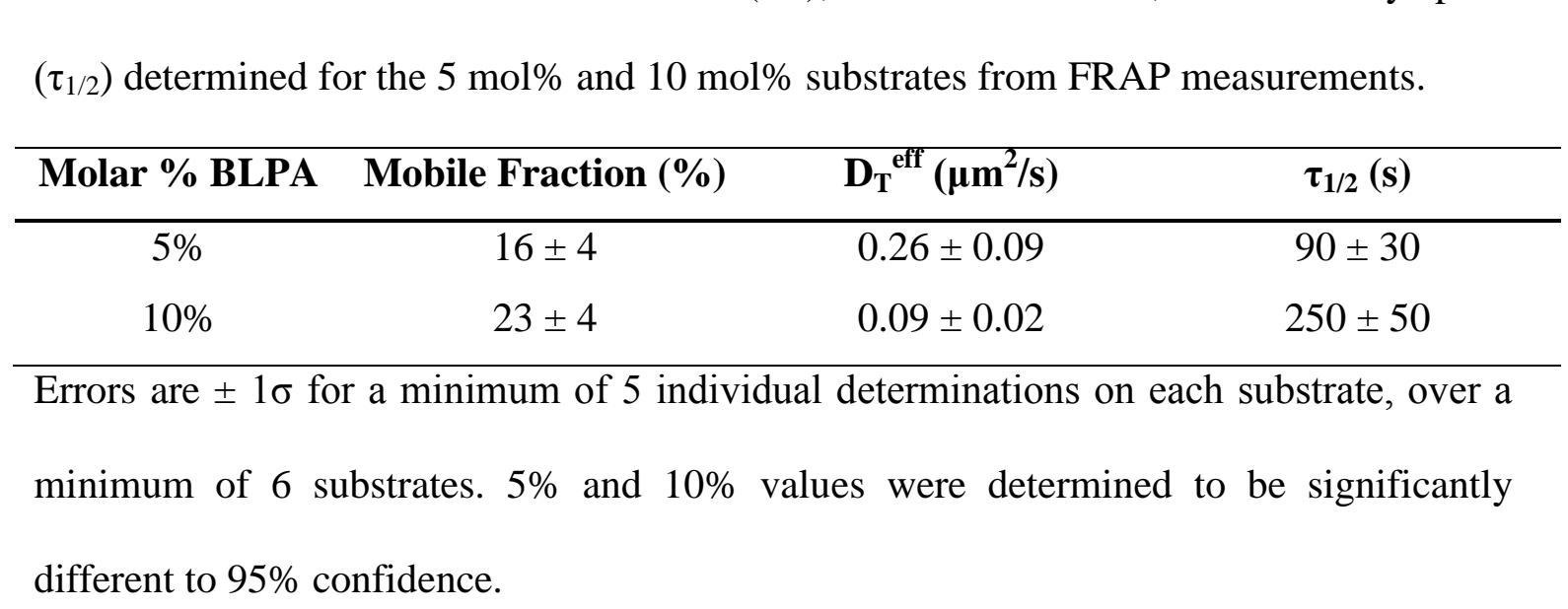

The authors gratefully acknowledge support for this work from The ACS Petroleum Research Fund under Grant 52692-ND6. We are also grateful to the National Science Foundation for providing support to TR through the REU program. 
Table 2: Reorientation time $\left(\tau_{\mathrm{HR}}\right)$, rotational diffusion constant $\left(\mathrm{D}_{\mathrm{ROT}}\right)$, and rotational wobbling constant $\left(\mathrm{D}_{\mathrm{W}}\right)$ determined by the local Environment of BLPA in a Ba-ODPA LB film.

\begin{tabular}{cccc}
\hline Molar \% BLPA & $\boldsymbol{\tau}_{\mathrm{HR}}(\mathbf{p s})$ & D $_{\text {ROT }}(\mathbf{M H z})$ & D $_{\mathbf{w}}(\mathbf{M H z})$ \\
\hline $5 \%$ & $3,400 \pm 220$ & $49.0 \pm 3.1$ & $30.9 \pm 2.0$ \\
$10 \%$ & $4,000 \pm 200$ & $41.8 \pm 2.1$ & $26.5 \pm 1.3$
\end{tabular}

Errors are $\pm 1 \sigma$ for a minimum of 2 individual scans over an area of $\mu \mathrm{m}^{2}$ on each substrate, over a minimum of 4 substrates. $5 \%$ and $10 \%$ rotational values were determined to be significantly different to $90 \%$ confidence ( $85 \%$ confidence for $\mathrm{H}$ Factor).

Table 3: Calculated Diffusion constant $\left(\mathrm{D}_{\mathrm{T}}\right)$, Unbound free fraction $\left(\mathrm{A}_{\text {free }}\right)$, Dissociation equilibrium constant $\left(\mathrm{K}_{\mathrm{eq}}\right)$, and Free energy for dissociation $\left(\Delta \mathrm{G}_{\mathrm{dis}}\right)$ for the BLPA in a Ba-ODPA LB film.

\begin{tabular}{ccccc}
\hline $\begin{array}{c}\text { Mole\% } \\
\text { BLPA }\end{array}$ & $\begin{array}{c}\mathbf{D}_{\mathbf{T}}{ }^{0} \\
\left(\mu \mathbf{m}^{2} / \mathbf{s}\right)\end{array}$ & $\begin{array}{c}\text { Unbound } \\
\text { fraction }\left(\boldsymbol{A}_{\text {free }}\right)\end{array}$ & $\mathbf{K}_{\mathbf{e q}}$ & $\begin{array}{c}\Delta \mathbf{G}_{\mathbf{d i s}} \\
(\mathbf{k J} / \mathbf{m o l})\end{array}$ \\
\hline $5 \%$ & $11.1 \pm 0.7$ & $0.023 \pm 0.009$ & $5.0(+4.9,-3.2) \times 10^{-4}$ & $18.5(+2.6,-1.7)$ \\
$10 \%$ & $9.6 \pm 0.5$ & $0.009 \pm 0.002$ & $8.2(+4,-3.3) \times 10^{-5}$ & $22.9(+1.3,-0.9)$ \\
\hline
\end{tabular}




\section{Literature Cited}

[1] M. Ratner, A brief history of molecular electronics, Nature Nanotech., 8 (2013) 378381.

[2] C.D. Bain, E.B. Troughton, Y.T. Tao, J. Evall, G.M. Whitesides, R.G. Nuzzo, Formation of Monolayer Films By the Spontaneous Assembly of Organic Thiols From Solution Onto Gold, J. Am. Chem. Soc., 111 (1989) 321-335.

[3] N. Camillone, C.E.D. Chidsey, P. Eisenberger, P. Fenter, J. Li, K.S. Liang, G.Y. Liu, G. Scoles, Structural Defects in Self-Assembled Organic Monolayers Via Combined Atomic-Beam and X-Ray-Diffraction, J. Chem. Phys., 99 (1993) 744-747.

[4] N. Camillone, C.E.D. Chidsey, G.Y. Liu, T.M. Putvinski, G. Scoles, SurfaceStructure and Thermal Motion of Normal-Alkane Thiols Self-Assembled On Au(111) Studied By Low-Energy Helium Diffraction, J. Chem. Phys., 94 (1991) 8493-8502.

[5] N. Camillone, C.E.D. Chidsey, G.Y. Liu, G. Scoles, Superlattice Structure At the Surface of a Monolayer of Octadecanethiol Self-Assembled On Au(111), J. Chem. Phys., 98 (1993) 3503-3511.

[6] C.E.D. Chidsey, G.Y. Liu, P. Rowntree, G. Scoles, Molecular Order At the Surface of an Organic Monolayer Studied By Low-Energy Helium Diffraction, J. Chem. Phys., 91 (1989) 4421-4423.

[7] C.E.D. Chidsey, G.Y. Liu, G. Scoles, J. Wang, Helium Diffraction From Overlayers Physisorbed On a Self- Assembled Organic Monolayer, Langmuir, 6 (1990) 1804-1806.

[8] C.E.D. Chidsey, M.D. Porter, D.L. Allara, Electrochemical Characterization of NAlkyl Thiol, Sulfide, and Disulfide Monolayers On Gold, J. Electrochem. Soc., 133 (1986) C130-C130.

[9] L.H. Dubois, B.R. Zegarski, R.G. Nuzzo, The Chemisorption of Organosulfur Compounds On Gold Surfaces - Construction of Well-Defined Organic-Solids, J. Vac. Sci. Technol. A-Vac. Surf. Films, 5 (1987) 634-635.

[10] L.H. Dubois, B.R. Zegarski, R.G. Nuzzo, Molecular Ordering of Organosulfur Compounds On $\mathrm{Au}(111)$ and $\mathrm{Au}(100)$ - Adsorption From Solution and in UltrahighVacuum, J. Chem. Phys., 98 (1993) 678-688.

[11] A. Ulman, Formation and structure of self-assembled monolayers, Chem. Rev., 96 (1996) 1533-1554. 
[12] D.S. Karpovich, G.J. Blanchard, Direct Measurement of the Adsorption Kinetics of Alkanethiolate Self-Assembled Monolayers on a Microcrystalline Gold Surface, Langmuir, 10 (1994) 3315-3322.

[13] H.M. Schessler, D.S. Karpovich, G.J. Blanchard, Quantitating the Balance between Enthalpic and Entropic Forces in Alkanethiol/Gold Monolayer Self Assembly, J. Am. Chem. Soc., 118 (1996) 9645-9651.

[14] D. Ryan, B.A. Parviz, V. Linder, V. Semetey, S.K. Sia, J. Su, M. Mrksich, G.M. Whitesides, Patterning multiple aligned seld-assembled monolayers using light, Langmuir, 20 (2004) 9080-9088.

[15] M.A. Rampi, O.J.A. Schueller, G.M. Whitesides, Alkanethiol self-assembled monolayers as the dielectric of capacitors with nanoscale thickness, Appl. Phys. Lett., 72 (1998) 1781-1783.

[16] H. Zhang, S.-W. Chung, C.A. Mirkin, Fabrication of sub-50-nm solid-state nanostructures on the basis of dip-pen nanolithography, Nano Lett., 3 (2003) 43-45.

[17] K.-B. Lee, E.-Y. Kim, C.A. Mirkin, S.M. Wolinsky, The use of nanoarrays for highly sensitive and selective detection of human immunodeficiency virus type 1 in plasma, Nano Lett., 4 (2004) 1869-1872.

[18] I. Langmuir, The constitution and fundamental properties of solids and liquids. II. Liquids, J. Am. Chem. Soc., 39 (1917) 1848-1906.

[19] K.B. Blodgett, I. Langmuir, Built-up films of barium stearate and their optical properties, Phys. Rev., 51 (1937) 964-982.

[20] K.B. Blodgett, Films built by depositing successive monomolecular layers on a solid surface, J. Am. Chem. Soc., 57 (1935) 1007-1022.

[21] K. Ariga, Y. Yamauchi, T. Mori, J.P. Hill, What can be done with the LangmuirBlodgett method? Recent developments and its critical role in materials science, Adv. Mat., 25 (2013) 6477-6512.

[22] M. Shimomura, K. Song, J.F. Rabolt, Spectroscopic determination of diffusion in Langmuir-Blodgett films. 1. Interlayer diffusion of cadmium arachidate and its deuterated analogue through a polymer interface., Langmuir, 8 (1992) 887-893.

[23] G.E. Fanucci, C.T. Seip, M.A. Petruska, C.M. Nixon, S. Ravaine, D.R. Talham, Organic/inorganic Langmuir-Blodgett films based on known layered solids: Divalent and trivalent metal phosphonates, Thin Solid Films, 327-329 (1998) 331-335.

[24] G.E. Fanucci, D.R. Talham, Langmuir-Blodgett films based on known layered solids: Lanthanide(III) octadecylphosphonate LB films, Langmuir, 15 (1999) 3289-3295. 
[25] D.R. Talham, Conducting and magnetic Langmuir-Blodgett films, Chem. Rev., 104 (2004) 5479-5501.

[26] H. Byrd, J.K. Pike, D.R. Talham, Inorganic monolayers formed at an organic template: A Langmuir-Blodgett route to monolayer and multilayer films of zirconium octadecylphosphonate, Chem. Mater., 5 (1993) 709-715.

[27] M.A. Petruska, D.R. Talham, Organic/inorganic Langmuir-Blodgett films based on metal phosphonates. 3. An azobenzene-derivatized phosphonic acid forms continuous lattice layers with divalent, trivalent, and tetravalent metal ions, Chem. Mater., 10 (1998) 3672-3682.

[28] S. Trabelsi, S. Zhang, Z. Zhang, T.R. Lee, D.K. Schwartz, Semi-fluorinated phosphonic acids form stable nanoscale clusters in Langmuir-Blodgett and selfassembled monolayers, Soft Matter, 5 (2009) 750-758.

[29] C.T. Seip, G.E. Granroth, M.W. Meisel, D.R. Talham, Langmuir-Blodgett films of known layered solids: Preparation and structural properties of octadecylphosphonate bilayers with divalent metals and characterization of a magnetic Langmuir-Blodgett film, J. Am. Chem. Soc., 119 (1997) 7084-7094.

[30] M.B. Forstner, J. Kas, D. Martin, Single lipid diffusion in Langmuir Monolayers, Langmuir, 17 (2001) 567-570.

[31] P. Bertoncello, I. Ciani, F. Li, P.R. Unwin, Measurement of apparent diffusion coefficients within ultrathin Nafion Langmuir-Schaefer films: Comparison of a novel scanning electrochemical microscopy approach with cyclic voltammetry, Langmuir, 22 (2006) 10380-10388.

[32] L.L. Wright, A.G.I. Palmer, N.L. Thompson, Inhomogeneous translational diffusion of monoclonal antibodies on phospholipid Langmuir-Blodgett films, Biophys. J., 54 (1988) 463-470.

[33] C. Selle, F. Ruckerl, D.S. Martin, M.B. Forstner, J. Kas, Measurement of diffusion in Langmuir monolayers by single particle tracking, Phys. Chem. Chem. Phys., 6 (2004) 5535-5542.

[34] M. Sickert, F. Rondolez, Shear viscosity of Langmuir monolayers in the low-density limit, Phys. Rev. Letters, 90 (2003) 126104-126101-126104.

[35] J. Ellenberg, E.D. Siggia, J.E. Moriera, C.L.P. Smith, J. F., H.J. Worman, J. Lippincott-Schwartz, Nuclear membrane dynamics and reassembly in living cells: Targeting of an inner nuclear membrane protein in interphase and mitosis, J. Cell Biol., 138 (1997) 1193-1206.

[36] I. Setiawan, G.J. Blanchard, Ethanol induced perturbations to planar lipid bilayer structures, J. Phys. Chem. B, 118 (2014) 537-546. 
[37] G. Lipari, A. Szabo, Effect of Librational Motion on Fluorescence Depolarization and Nuclear Magnetic Resonance Relaxation in Macromolecules and Membranes, Biophys. J., 30 (1980) 489-506.

[38] A. Szabo, Theory of fluorescence depolarization in macromolecules and membranes, J. Chem. Phys., 81 (1984) 150-167.

[39] Langmuir-Blodgett Films, Springer Science, New York, 1990.

[40] J.F. Rabolt, C.F. Burns, N.E. Schlotter, J.D. Swalen, Anisotropic orientation in molecular monolayers by infrared spectroscopy, J. Chem. Phys., 78 (1983) 946-952.

[41] T.A. Sherstneva, K.P. Birin, V.V. Arslanov, The aggregation behavior of amphiphilic pyrene chromophore in solutions and langmuir monolayers, Fizikokhimiya Poverkhnosti i Zashchita Materialov, 49 (2013) 72-87.

[42] S. Varughese, Non-covalent routes to tune the optical properties of molecular materials, J. Mater. Chem. C, 2 (2014) 3499-3516.

[43] G. Pescitelli, L. DiBari, N. Berova, Application of electronic circular dichroism in the study of supramolecular systems, Chem. Soc. Rev., 43 (2014).

[44] F.C. Spano, C. Silva, H- and J-Aggregate behavior in polymeric semiconductors, Annu. Rev. Phys. Chem., 65 (2014) 477-500.

[45] H. Lu, Z. Xue, J. Mack, Z. Shen, X. You, N. Kobayashi, Specific $\mathrm{Cu}^{2+}$-induced Jaggregation and $\mathrm{Hg}^{2+}$-induced fluorescence enhancement based on BODIPY, Chem. Commun., 46 (2010) 3565-3567.

[46] S. Choi, J. Bouffard, Y. Kim, Aggregation-induced emission enhancement of a meso-trifluoromethyl BODIPY via J-aggregation, Chem. Sci., 5 (2014) 751-755.

[47] Y. Tokoro, A. Nagai, Y. Chujo, Nanoparticles via H-aggregation of amphiphilic BODIPY dyes, Tet. Letters, 51 (2010) 3451-3454.

[48] J.-H. Olivier, J. Widmaier, R. Ziessel, Near-infrared fluorescent nanoparticles formed by self-assembly of lipidic (Bodipy) dyes, Chem. Eur. J., 17 (2011) 11709-11714.

[49] R. Metzler, J. Klafter, The random walk's guide to anomalous diffusion: A fractional dynamics approach, Physics Reports, Elsevier, Amsterdam, 2000, pp. 1-77.

[50] M. Palombo, A. Gabrielli, V.D.P. Servedio, G. Ruocco, S. Capuani, Structural disorder and anomalous diffusion in random packing of spheres, Sci. Rep., 3 (2013) 1-7.

[51] D.S. Novikov, E. Fieremans, J.H. Jensen, J.A. Helpern, Random walks with barriers, Nature Phys., 7 (2011) 508-514. 
[52] S.J. Suresh, V.M. Naik, Hydrogen bond thermodynamic properties of water from dielectric constant data, J. Chem. Phys., 113 (2000) 9727-9732.

[53] H.Y. Nie, Revealing different bonding modes of self-assembled octadecylphosphonic acid monolayers on oxides by time-of-flight secondary ion mass spectrometry: Silicon vs Aluminum, Anal. Chem., 82 (2010) 3371-3376.

[54] K.A.T. Silverstein, A.D.J. Haymet, K.A. Dill, The strength of hydrogen bonds in liquid water and around nonpolar solutes, J. Am. Chem. Soc., 122 (2000) 8037-8041.

[55] E.N. Rizkalla, M.T.M. Zaki, Metal chelates of phosphonate-containing ligands 1. Stability of some $\mathrm{N}, \mathrm{N}, \mathrm{N}^{\prime}, \mathrm{N}^{\prime}$-ethylenediaminetetra(methylenephosphonic) acid metal chelates, Talanta, 26 (1979) 507-510.

[56] E.N. Rizkalla, M.T.M. Zaki, Metal chelates of phosphonate-containing ligands VI. Complexes of ethylenediaminetetra(methylenephosphonic) acid with $\mathrm{Cd}, \mathrm{Mg}, \mathrm{Ca}$ and $\mathrm{Ba}$, Talanta, 27 (1980) 769-770.

[57] E. Barouda, K.D. Demadis, S.R. Freeman, F. Jones, M.I. Ogden, Barium sulface crystallization in the presence of variable chain length aminomethylenetetraphosphonates and cations $\left(\mathrm{Na}^{+}\right.$or $\left.\mathrm{Zn}^{2+}\right)$, Crystal Growth Des., 7 (2007) 321-327.

[58] G.J. Blanchard, A study of the state-dependent reorientation dynamics of oxazine 725 in primary normal aliphatic alcohols, Journal of Physical Chemistry, 92 (1988) 63036307.

[59] E. Blomgren, J.O.M. Bockris, C. Jesch, The adsorption of butyl, phenyl and naphthyl compounds at the interface mercury-aqueous acid solution, J. Phys. Chem., 65 (1961) 2000-2010.

[60] D.S. Karpovich, G.J. Blanchard, Dynamics of a tethered chromophore imbedded in a self-assembled monolayer, Langmuir, 12 (1996) 5522-5524. 


\section{Figure Captions}

Figure 1: Structures of the components used in the formation of the LB film: (A) ODPA, (B) TopFluor Lyso PA (BLPA)

Figure 2: $\pi / \mathrm{A}$-isotherm of ODPA on an aqueous subphase with a $\mathrm{Ba}^{2+}$ concentration of 5 $\mathrm{mM}$ (i.) and an aqueous subphase (ii.). $\pi / \mathrm{A}$-isotherm of BLPA on an aqueous subphase (iii.) and with a $\mathrm{Ba}^{2+}$ concentration of $5 \mathrm{mM}$ (iv.). The temperature for the subphase was $23^{\circ} \mathrm{C}$ and $\mathrm{pH}=5.4$. The monolayers were compressed at a rate of $0.8 \AA^{2} / \mathrm{min}$. Yellow circles represent pressures at which BAM images (B-G) were taken. (A) Images B, D, and $\mathrm{F}$. are of ODPA on an aqueous subphase containing $5 \mathrm{mM} \mathrm{BaCl}_{2}$, while images $\mathrm{C}, \mathrm{E}$, and G. are of ODPA on an aqueous subphase. Scale bars represent $100 \mu \mathrm{m}$.

Figure 3: $\pi /$ A-isotherm of the two component monolayers containing ODPA and BLPA on an aqueous subphase with a $\mathrm{Ba}^{2+}$ concentration of $5 \mathrm{mM}$, and $0 \mathrm{~mol} \%$ BLPA (i.), 5 mol\% (ii.), and $10 \mathrm{~mol} \%$ (iii.). The temperature for the subphase was $23^{\circ} \mathrm{C}$ and $\mathrm{pH}=5.4$. The monolayers were compressed at a rate of $0.8 \AA^{2} / \mathrm{min}$. Yellow circles represent pressures at which BAM images (B-G) were taken (A). Images at 0 mol\% BLPA are presented in Fig. 2, Images B, D, and F are of ODPA and 5 mol\% BLPA, and images C, E, and G. are of monolayers of ODPA and 10 mol\% BLPA. Scale bars represent $100 \mu \mathrm{m}$.

Figure 4: Schematic representation of a structural description of $\mathrm{H}$ - and J-aggregates of the BODIPY chromophore (A). Emission spectra of two component LB films of BLPA5 (blue), and BLPA-10 (green). Absorbance and emission spectra of BLPA in THF 
(black). All spectra were normalized to unity (B). Steady state fluorescence images of BLPA-5 are shown in (C) and BLPA-10 in (D). Scale bars represent $500 \mu \mathrm{m}$.

Figure 5: FRAP recovery of the BLPA chromophore in BLPA-5 (blue) and BLPA-10 (green). The orange lines of inset represent best-fit lines using the Ellenberg equation. Both FRAP curves were normalized prior to fit.

Figure 6: Typical experimental anisotropy decay functions for the BLPA-5 LB films are shown in A, and BLPA-10 in B. The solid orange line is the best-fit decay, and the residuals are plotted below. Fluorescence transients were fit to determine the fluorescence lifetime shown in $\mathrm{B}$ and $\mathrm{C}$. The colors shown represent the lifetime value at a given pixel, with blue being short lifetimes and red being longer lifetimes. Fluorescence anisotropy decay time constant images are shown in D and E. The colors shown are set by the imaging software, with blue being short decay time constants and red being longer decay time constants. All images were acquired using a 10x microscope objective. Images $\mathrm{C}$ and $\mathrm{E}$ represent BLPA-5, images $\mathrm{D}$ and $\mathrm{F}$ represent BLPA-10. Scale bars represent $500 \mu \mathrm{m}$.

Figure 7: Schematic of equilibrium system under consideration. 


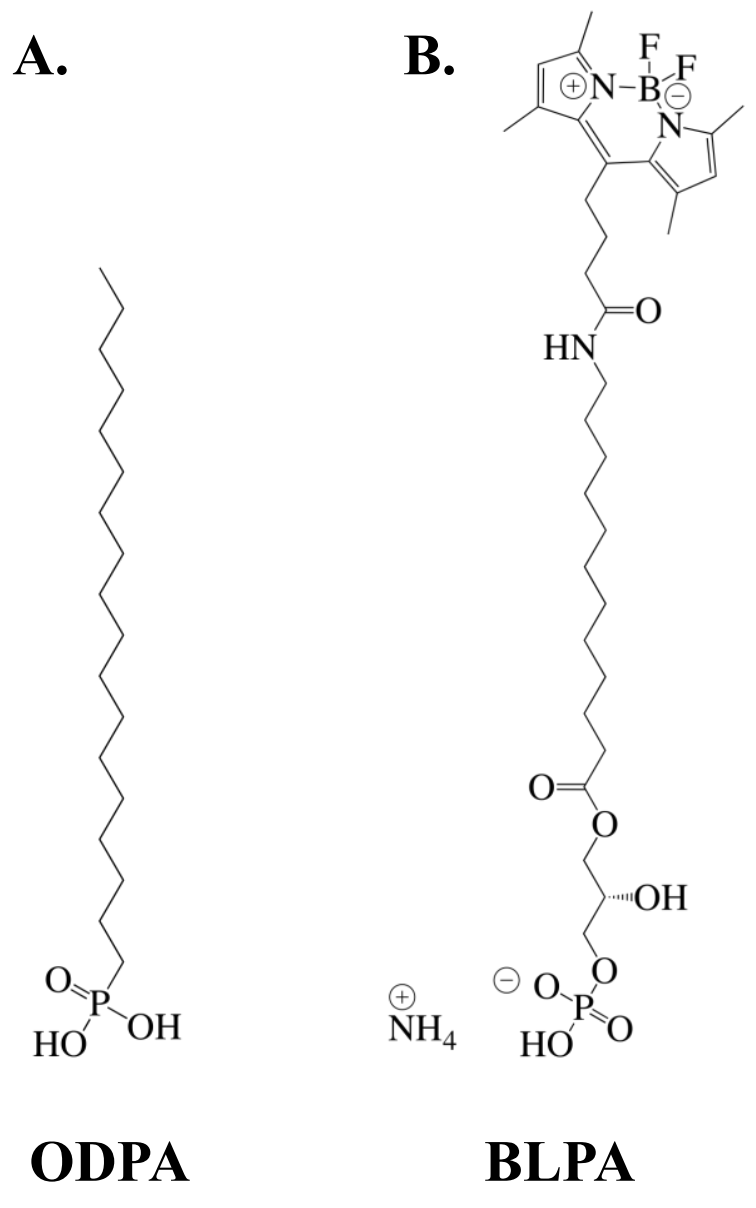

Baumler, Reidy, and Blanchard, Figure 1

40

41

42

43

44

45

46

47

48

49

50

51

52

53

54

55

56

57

58

59

60

61

62 

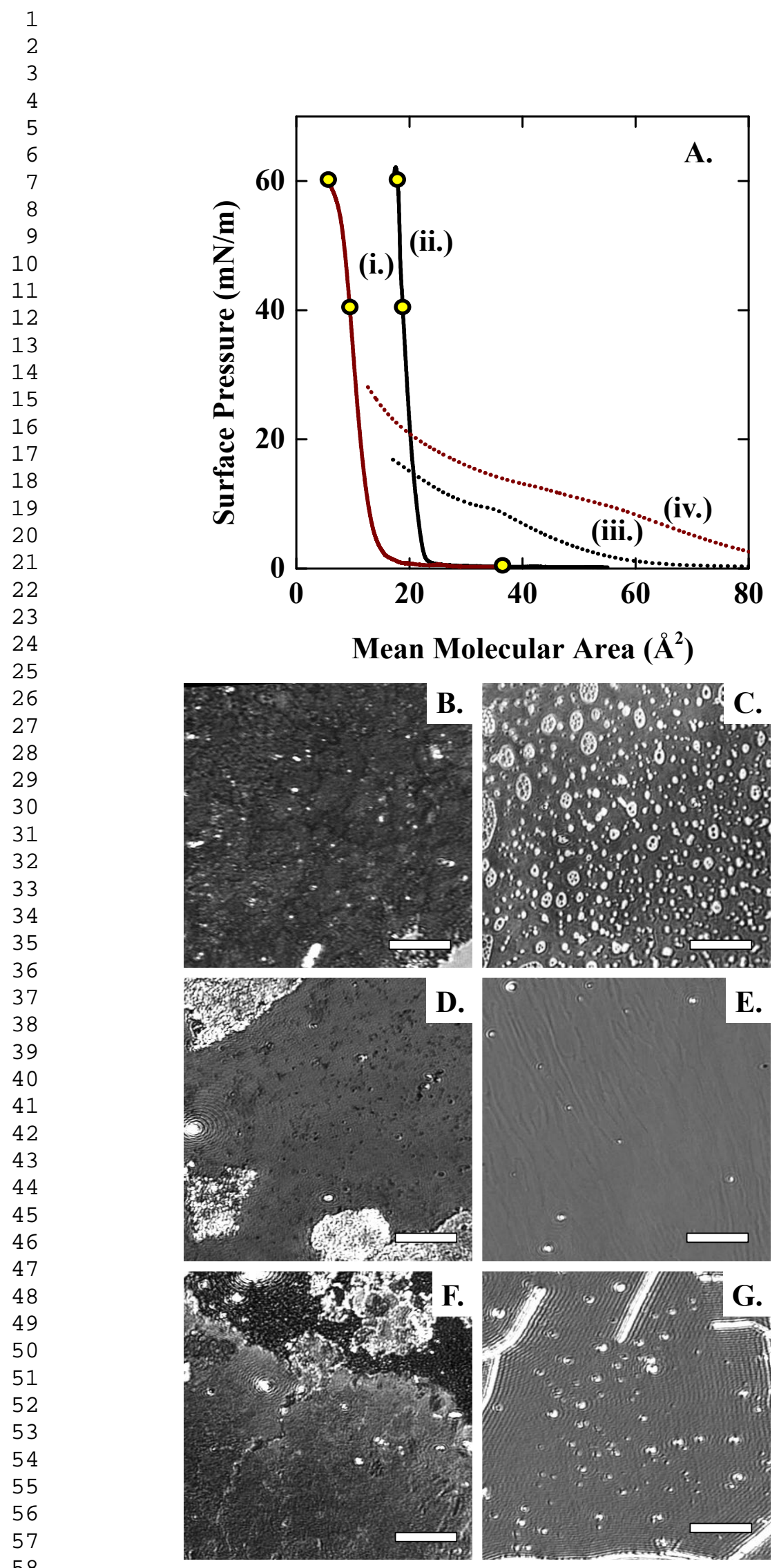

Baumler, Reidy, and Blanchard, Figure 2 

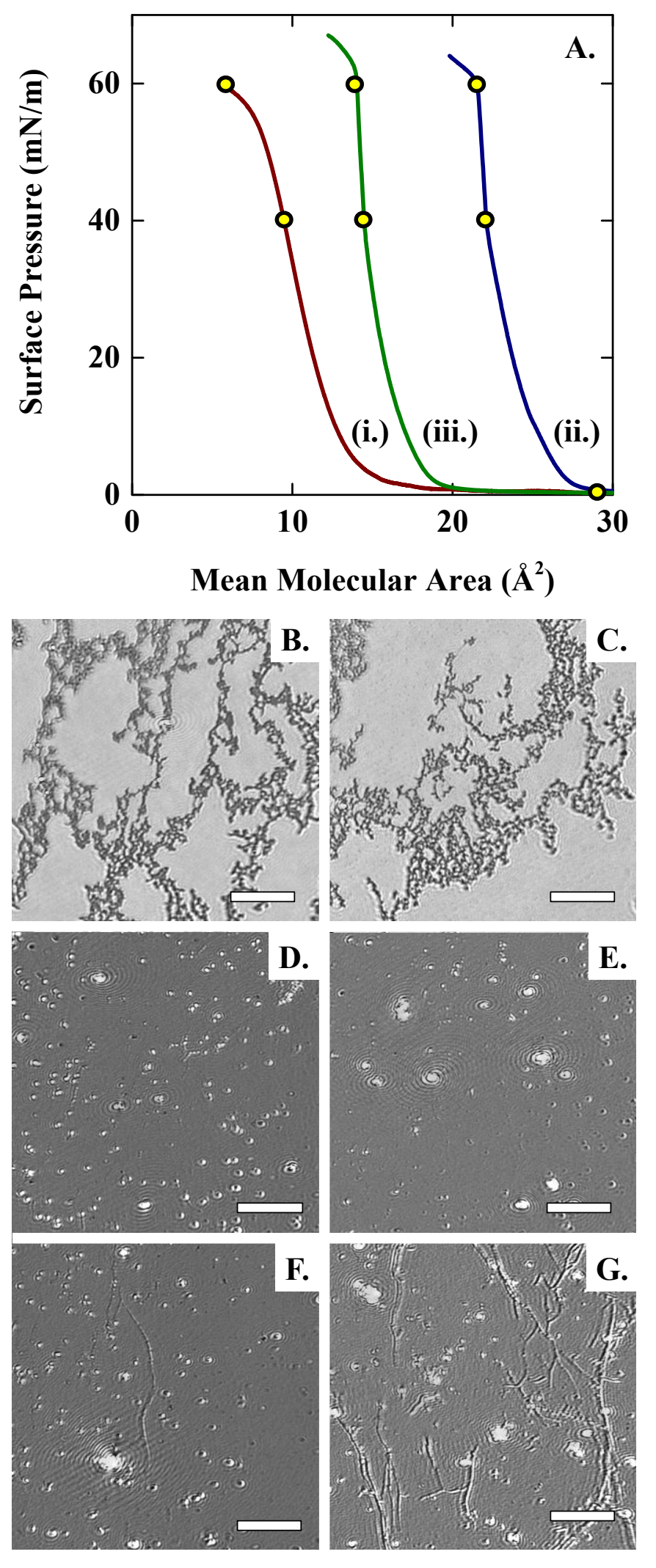

Baumler, Reidy, and Blanchard, Figure 3 

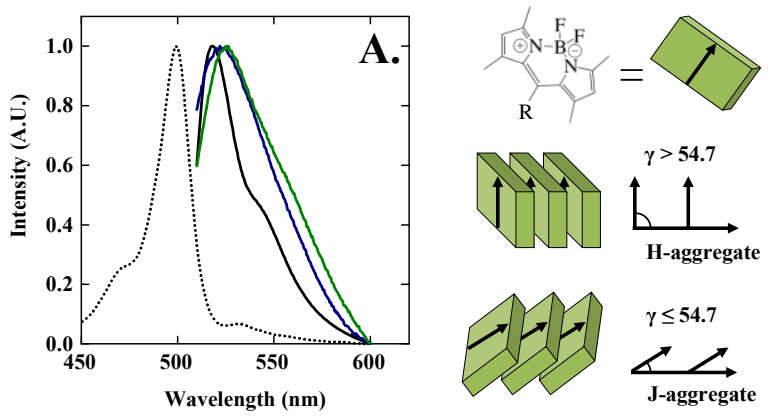

B.
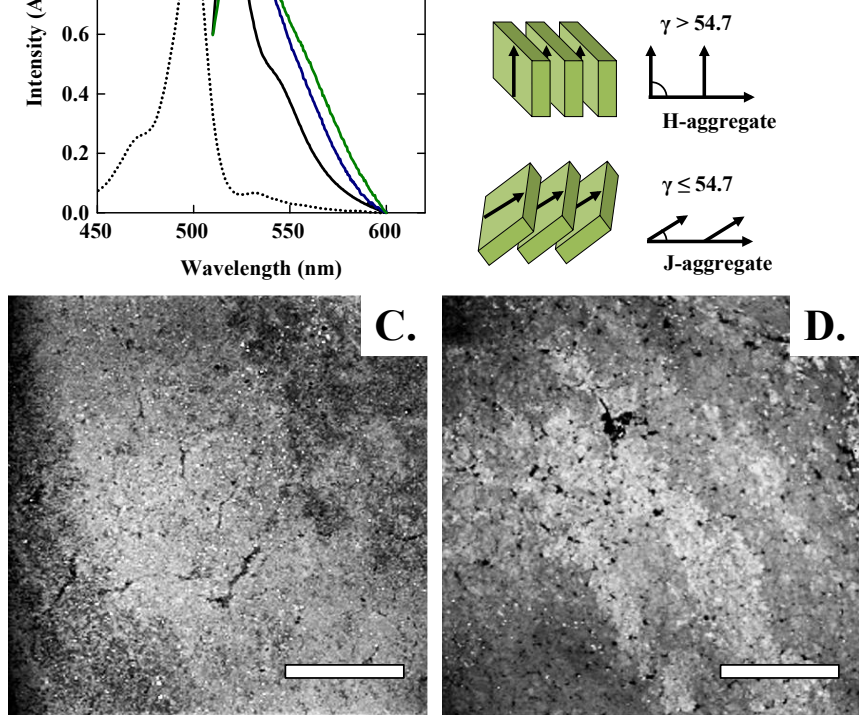

Baumler, Reidy, and Blanchard, Figure 4 


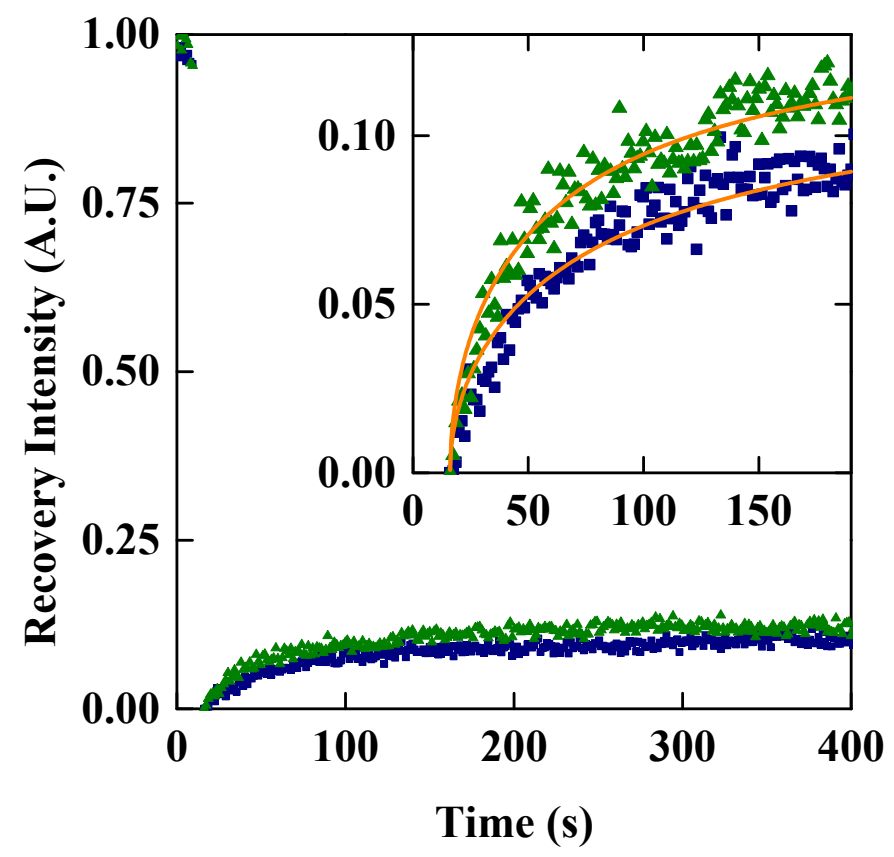

Baumler, Reidy, and Blanchard, Figure 5 

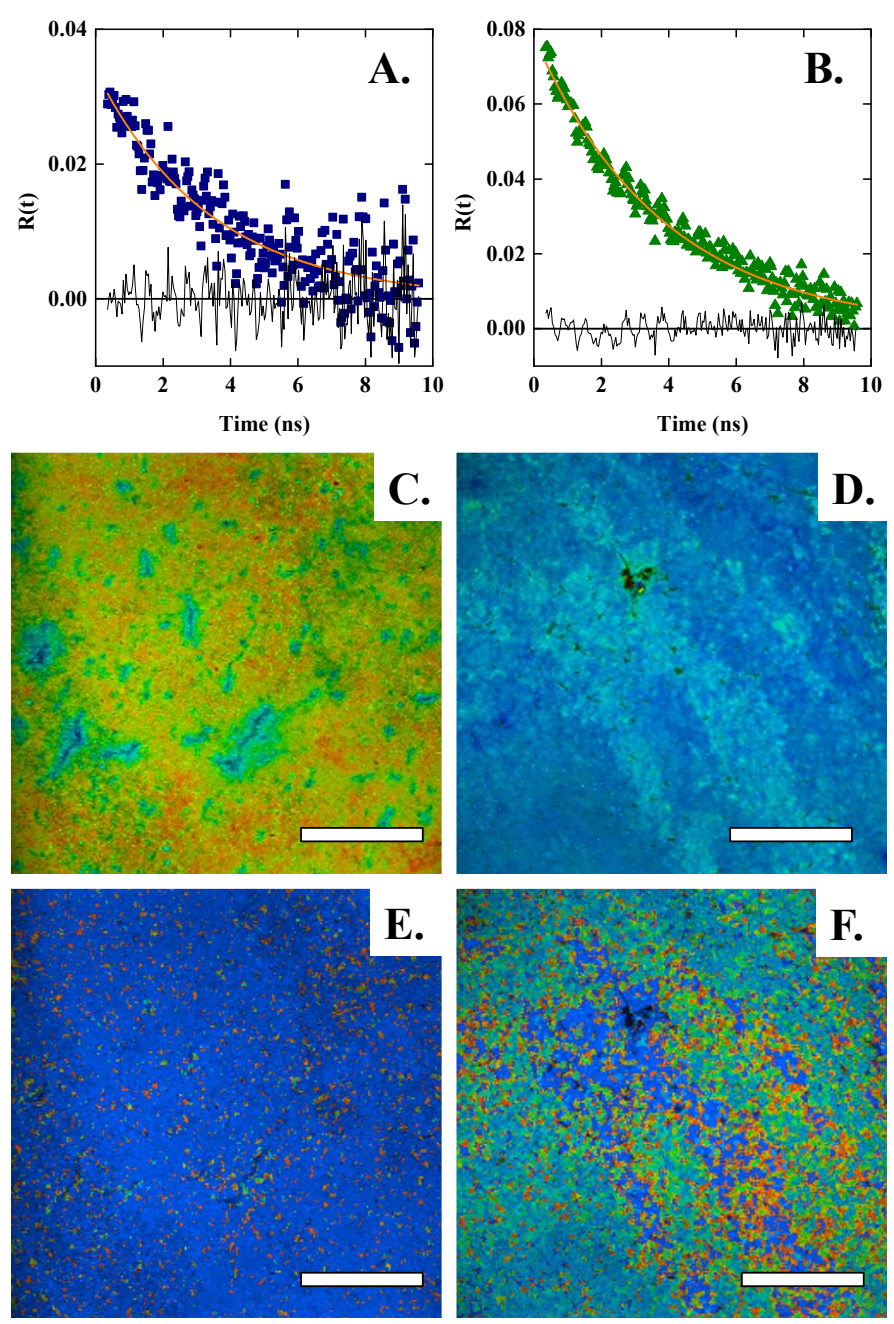

Baumler, Reidy, and Blanchard, Figure 6 


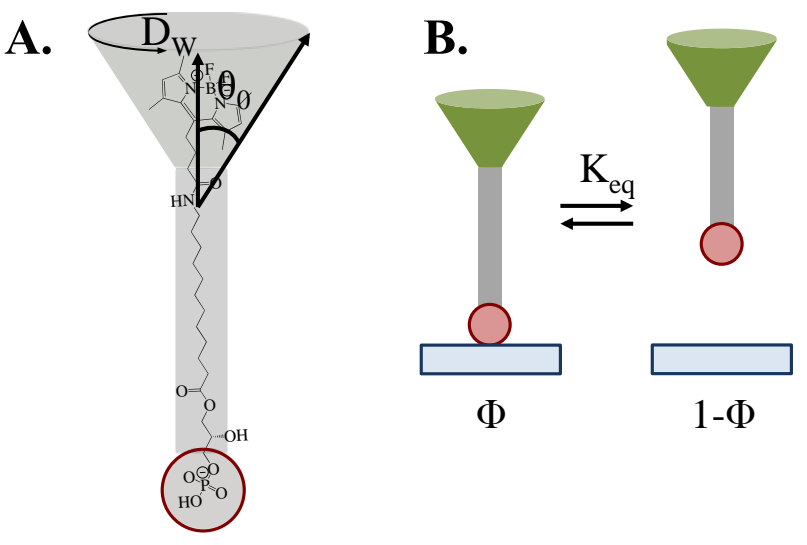

Baumler, Reidy and Blanchard, Figure 7

24

25

26

27

28

29

30

31

32

33

34

35

36

37

38

39

40

41

42

43

44

45

46

47

48

49

50

51

52

53

54

55

56

57

58

59

60

61

62

63 


\section{Measure Diffusional Motions \\ Quantitative Gauge of Film Properties}
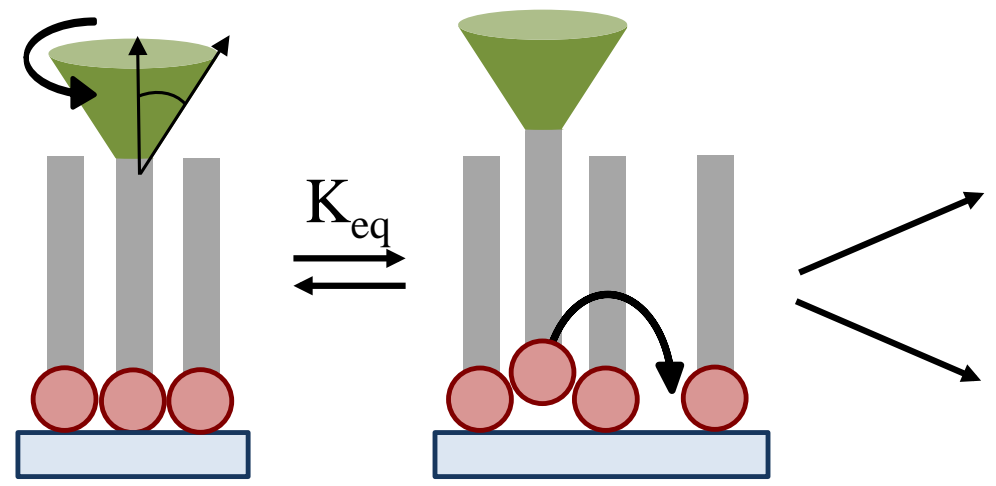

Rotational Diffusion + Translational Diffusion 